\title{
SPECTROSCOPIC IMPLICATIONS OF NEW HOLOGRAPHIC IMAGING METHODS
}

\author{
by GEORGE W. STROKE
}

The University of Michigan, Ann Arbor, Michigan, U.S.A.

\section{Synopsis}

Significant increases in luminosity, detection and resolution capabilities may result from extending to spectroscopic and astronomical instruments some of the new advances, recently made in the field of wavefront-reconstruction imaging (holography), first described by D. Gabor in 19481) 2) 3) 4).

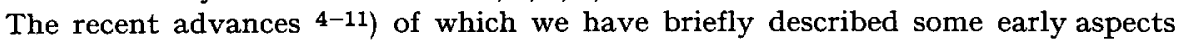
elsewhere have already permitted us to obtain spectra in a holographic Fouriertransforming ${ }^{7}$ ) ${ }^{8}$ ) arrangement, using no scanning in the interferometer, and displaying the spectra by optical Fourier-transform reconstruction from the interferometric hologram ${ }^{9}$ ), rather than by digital computation. In another work, we have now been able to holographically compensate a posteriori for the "slit spreadingeffect", in a coherent-light imaging system, and to retrieve the resolution by a correlation-reconstruction method 10) 11). "Erasing" of selected image portions, by actually adding the complex amplitudes in two images, $180^{\circ}$ out of phase, in a holographic arrangement has also been achieved ${ }^{12}$ ) and may be used for increasing detection of selected image portions in astronomical and spectroscopic plates. Previously unpublished advances and some details of the new holographic imaging methods are given.

Dedication. One hundred years, since the birth of Pieter Zeeman, and just over six decades following the 1902 award of the Nobel Prize to him, jointly with Hendrik Antoon Lorentz, another of the great Dutchmen, for providing not only one of the most powerful tools for the observation of the atomic structure of matter, but also for cementing a cornerstone in one of the most direct proofs of the electromagnetic character of light, it may be fitting to discuss some strange new phenomena in the formation of images, by light, and som new possibilities which now appear, in our efforts to further look into the nature of matter, by spectroscopy, and perhaps into the origins of biological life itself, by image-forming, holographic $X$-ray microscopy.

Introduction. It has recently become evident that the field of holography, (loosely called "lensless photography" or "wavefront reconstruction photography") has much wider, and perhaps much more extensive ramifications 
in optics, than might have appeared from its original name of "wavefrontreconstruction imaging", $\left.{ }^{12}{ }^{2}\right)^{3}$ ) or indeed, more recently from the achievements of "three-dimensional photography", which were first reported in 1964 and 1965 by E. N. Leith, J. U patnieks and G. W. Stroke4) 13) 14) 15).

A hologram (loosely called "lensless photograph") is basically a twobeam interferogram, $\left.{ }^{4}\right)^{13}{ }^{14}$ ) formed by making a plane or a spherical wave interfere with the light scattered from an object or a transparency, in such a way that an image can later be reconstructed from the hologram, with or without the intervention of any focusing lenses or mirrors. More generally, it has been shown (by Gabor, Stroke, e.a. ${ }^{12}$ )) that holograms can be formed by a successive superposition (in the latent photographic "image") of the intensities of several interferograms, and be used for image synthesis and filtering methods long known in electronics. For example, actual image "erasing" has been achieved by Gabor, Stroke, e.a.12) by interferometrically adding the intensities in two images, with a phase shift of $180^{\circ}$ between them!

Because of these, and other, new and sophisticated ramifications of this new field of optics, it has recently become known as holography, a name given to it on November 9, 1964, by the author to mark the basic contribution of its originator, Prof. D. Gabor $\left.\left.{ }^{1}\right)^{2}\right)^{3}$ ) who coined the word "hologram" in the first place, in 1948, so as to indicate that the "whole" imageforming information in the field, to be photographed, can be recorded on a photographic plate, placed in front of a camera lens, rather than in its focal plane, where it generally belongs.

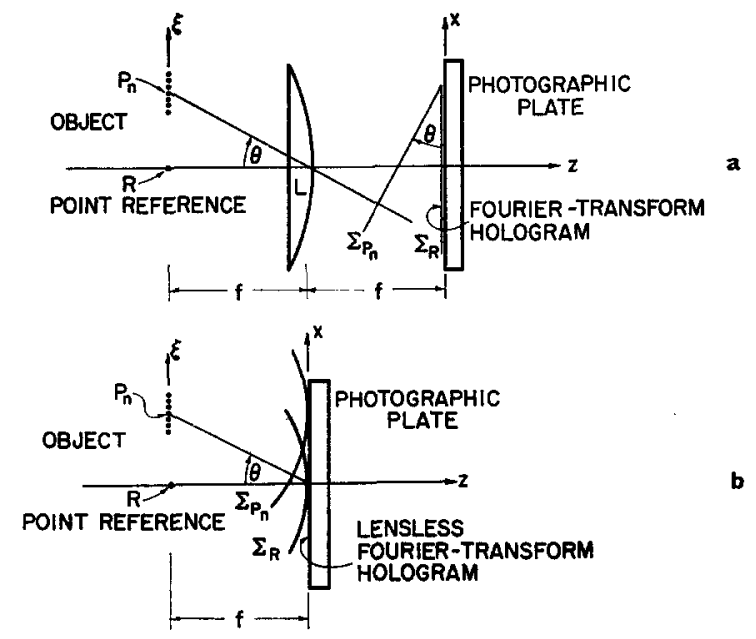

Fig. 1. Fourier-transform hologram recording arrangements.

a. Arrangement using lens.

b. "Lensless"'Fourier-transform hologram recording arrangement (according to Stroke, loc. cit. 7). 


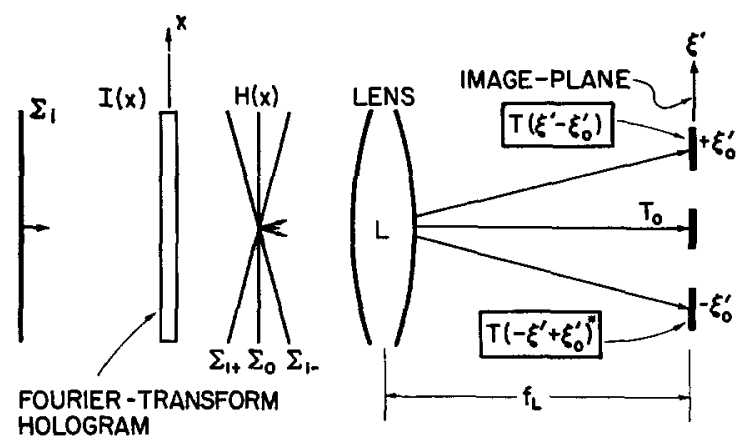

Fig. 2. Fourier-transform reconstruction from Fourier-transform holograms.5) 23)

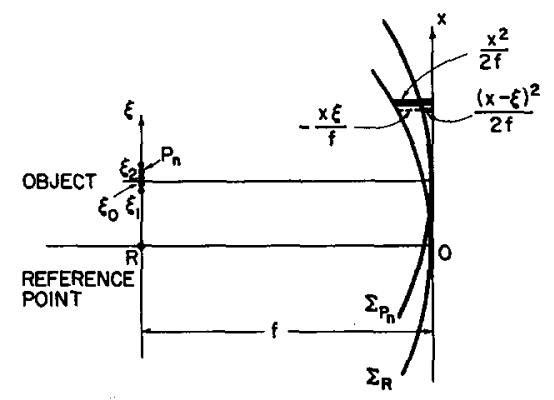

Fig. 3. Fourier-transform hologram recording parameters. $\Sigma_{P_{n}}=$ wavefront scattered by object point $P_{n}$. $\Sigma_{R}=$ wavefront scattered by reference point $R$.

The principles and new developments in holography, have been recently reviewed by a number of authors (see e.g. ref. 4, 13-15) and will not be repeated here. A considerable theoretical and experimental simplification has resulted in holography from the use of "Fourier-transform holography" principles and arrangements, first introduced by the author in 196313) (see also refs. 4-14).

The two basic Fourier-transform hologram recording arrangements are illustrated in fig. 1, and a Fourier-transform image reconstruction arrangement in fig. 2. Hologram recording parameters are shown in fig. 3. A basic advantage of Fourier-transform holograms (compared to Fresnel transform holograms) is their considerably greater resolution capability, first pointed out by the author in 19644-8) ${ }^{10}$ ).

Because of the possible implications which two aspects of the recent holographic advances may have on spectroscopy, and indeed in astronomical imaging, we now give some previously unpublished details of our method of holographic Fourier-transform spectroscopy, and of our method of $a$ posteriori "resolution-retrieving" image compensation or "decoding". 
1. Holographic Fourier-transform spectroscopy ${ }^{9}$ ). The remarkable properties and the important advantages of several forms of "Fourier-transform spectroscopy" have now been established ${ }^{16-21}$ ), following initial work by Jacquinot ${ }^{19}$ ), Fellgett ${ }^{20}$ ), Strong ${ }^{21}$ ), and others. Among the principal advantages are a simultaneous recording of all spectral elements (recording time independent of the spectral width) and high luminosity. The method requires very accurate (ruling-engine quality) moving-mirrormotion (or scanning) and computation (by Fourier transformation) of the spectrum from the photoelectrically recorded interferogram. The recent advances in holographic (wavefront-reconstruction) imaging, and in its Fourier-transform formulation have made it reasonable to investigate possible simplifications which might result from extensions of holography to such applications as spectroscopy and astronomy.

The theoretical principles and experimental verifications of a holographic method of "Fourier-transform spectroscopy" were first demonstrated in our ref. 9. The method permits one to obtain the spectrum without any computation, and indeed in an interferometric system having completely

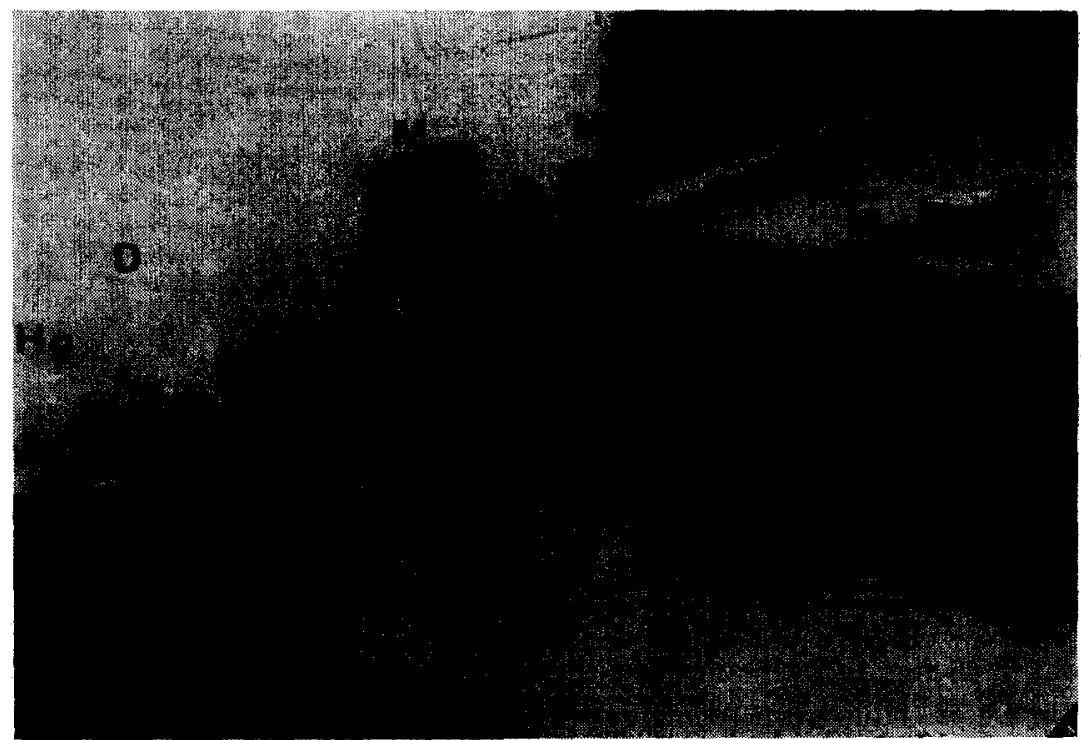

Fig. 4.* Interferometer used for recording spectroscopic Fourier-transform holograms with a spectrally noncoherent source (according to Stroke and Funkhouser, loc. cit. 9). $\mathrm{Hg}=$ cold mercury arc, $\mathrm{D}=$ diffusing glass, $\mathrm{M}, \mathrm{M}=$ interferometer mirrors, $\mathrm{B}=$ beam splitter, $\mathrm{H}=$ hologram photographic plate.

*) Note added in proof: Recent experiments (March 1966) by Stroke and Funkhouser have shown certain advantages of "triangular-path" compensated "Sagnac-type" interferometers for this work (unpublished), (22). 
stationary elements and medium. Various methods for transcribing onto film the photoelectric recordings of the Fourier transforms (or indeed Fresnel transforms) of spectra have been suggested for the purpose of optically reconstructing the spectrum. In our method, no transcription of a photoelectrically recorded spectrum, nor indeed any interferometer scanning are required.

As a model, we may discuss the recording of the spectral Fourier-transform hologram in a two-beam interferometer, in which the wavefronts form a small angle $\theta$ with each other (See fig. 4). The interference-fringe system photographically recorded in a plane "parallel" to the bisector of the wavefronts is proportional (in the holographic sense) to a non-coherent superposition of the monochromatic fringe systems corresponding to each wavelength $\lambda$. As we show below the equation of the fringe system in the plane of the photographic plate is readily found to be equal to

$$
I(x)=\int_{0}^{\infty} I(\sigma)[1+\cos 2 \pi \sigma \theta x] \mathrm{d} \sigma
$$

where $\sigma=1 / \lambda(\mathrm{cm})$ and $I(\sigma)$ is the spectral intensity distribution (the spectrum) of the source. Eq. (1), the cosine Fourier transform of $I(\sigma)$ is recognized as the equation on the spectral Fourier transform hologram of $I(\sigma)$ (i.e. a "noncoherent" superposition of sinusoidal intensity gratings, one per each $\lambda$ ). Consequently, illumination of the hologram by a spatially coherent, monochromatic plane wave (as in fig. 2) will produce in the focal plane of a lens the spectrum $I(\sigma)$, symmetrically displayed on the two sides of the optical axis (one pair of spectral lines for each grating recorded, plus zero-frequency terms).

The compensated Michelson-Twyman-Green interferometer used in our experiments ${ }^{9}$ ) to record the hologram is again shown in fig. 4, a hologram of the spectrum of a cold mercury arc, in fig. 5 and a reconstruction of the spectrum obtained by illuminating the hologram in $6328 \AA$ laser light (See fig. 2) is also again in fig. 6 for the purpose of clarity of our discussion. A very wide, diffusely illuminated source "aperture" was used in the recording, with an interferometer wedge of about 30 "white-light (mercury)" fringes per millimeter, near zero-path difference, and with Kodak $649 \mathrm{~F}$ high resolution plates. (Aligning experiments were carried out on Polaroid P/N film). Initial experiments with a flash-light source have shown that a continuous spectrum does produce a recordable interferometric hologram, and a reproducible spectrum).

Although eq. (1) can be readily obtained almost by inspection ${ }^{13}$ ) ${ }^{14}$ ) (see also ref. 16), it may be in order to give some of the steps of a more detailed analysis.

Let $E(x, y, z)$ be the electric field in a plane wave of wavelength $\lambda$, travelling at an angle $(\theta / 2)$ with respect to the $z$ axis in the interferometer, such 
that (in an $x$ plane, normal to the $z$ axis)

$$
E(x, y)=E_{0} \exp [2 \pi i \sigma x \sin (\theta / 2)+\omega t]
$$

where $\sigma=1 / \lambda, \omega=2 \pi t, c=t \lambda=$ velocity of light and $t=$ time.

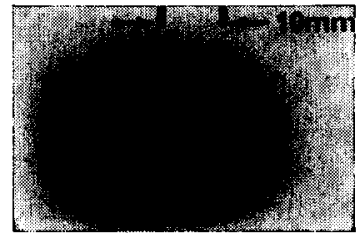

Fig. 5. Spectroscopic Fourier-transform hologram recorded in interferometer of Fig. 4.

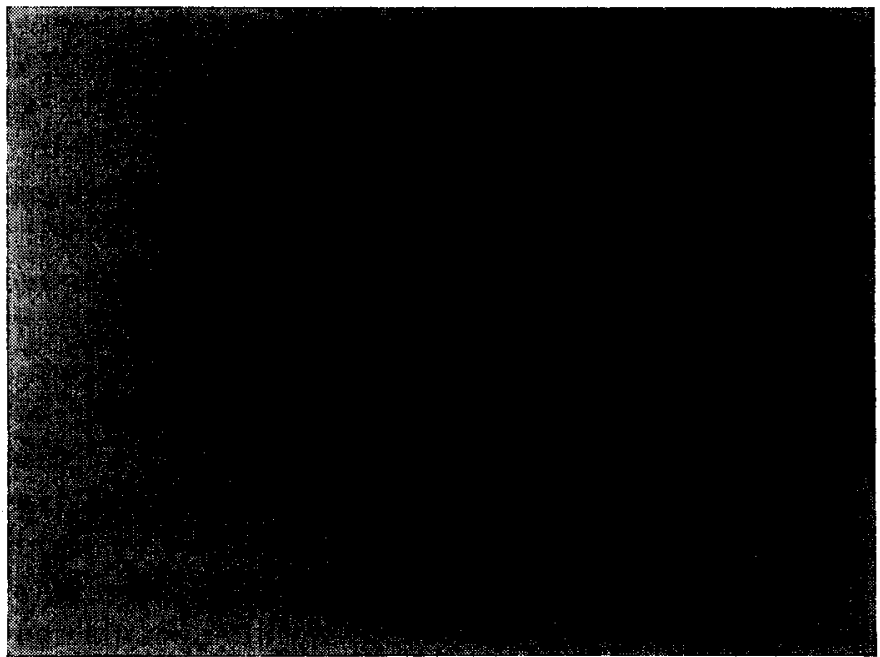

Fig. 6. Fourier-transform reconstruction of mercury spectrum, obtained in the focal plane of a lens (see Fig. 2) by projecting a plane wave of $6328 \AA$ laser light through the spectroscopic Fourier-transform hologram of Fig. 5. Note the characteristic symmetrical display of the spectrum, into a left and right "side band", which permits wavelength determination by a simple measurement of distance ratios. Identified, in the left side band are the mercury lines 1 ): $v(3663 \AA), b(4047 \AA, 4078 \AA), g(4358 \AA), y(5461 \AA)$, $r(5770 \AA, 5790 \AA)$ 
Let a second plane wave of wavelength $\lambda$, traveling at the angle $(-\theta / 2)$ with respect to the $z$ axis be such, that in the $x$ plane we have

$$
E(x, y)=E_{1} \exp [2 \pi i \sigma x \sin (-\theta / 2)+\omega t+\phi]
$$

where $\phi$ is a phase difference. When the two waves of eq. (2) and of eq. (3) are made to interfere on a photographic plate, in the $x$ plane, the resultant intensity, recorded on the plate, is

$$
[I(x, y)]_{\lambda}=\frac{1}{2} E_{0}^{2}+\frac{1}{2} E_{1}^{2}+E_{1} E_{0} \cos [2 \pi \sigma x \sin (\theta / 2)-\phi]
$$

We may now arrange the interferometer, so as to record the interference pattern at zero-path difference, for which $\phi=0$. We may further simplify the analysis by assuming $E_{0}^{2}=E_{1}^{2}=I(\sigma)$, where $I(\sigma)$ is the intensity of the source at the wave number $\sigma$, and also let $\theta$ be a small angle. Under these simplifying assumptions, we readily obtain from eq. (4) the equation

$$
[I(x, y)]_{\lambda}=I(\sigma)[1+\cos 2 \pi \sigma \theta x]
$$

which is the intensity of the interference fringe pattern (i.e. hologram) recorded in a bandwidth $\Delta \sigma$, centered at the wave number $\sigma$.

Because of the noncoherence between spectral elements (characteristic of sources generally studied by Fourier-transform spectroscopy, see e.g. ref. 16), the interferogram (i.e. hologram) formed when the interferometer is illuminated by polychromatic source, having an intensity - distribution $I(\sigma)$, it immediately follows that the intensity distribution $I(x)$ in the hologram will be given by

$$
I(x)=\int_{0}^{\infty} I(\sigma)[1+\cos 2 \pi \sigma \theta x] \mathrm{d} \sigma
$$

which is indeed identical to eq. (1).

We have previously shown (see e.g. our ref. $4,5,14$ ) that the complex amplitude $H(x)$ transmitted through a hologram, on which we have recorded an intensity $I(x)$, may be given by an equation of the form

$$
H(x) \cong I(x)^{-\gamma / 2}
$$

where $\gamma=$ gamma of the photographic plate. Under the usual holographic conditions, when $I(x)$ may be wirtten in the form

$$
I(x) \cong 1+\varepsilon
$$

we obtain, by a series expansion, the equation

$$
H(x) \cong 1-\gamma / 2 \varepsilon
$$

which shows the remarkable fact that the complex amplitude in the terms of interest in $H(x)$, [for Fourier-transform holography reconstruction], is linearly related to the recorded intensity under these conditions, and the 
reconstructed image intensity is independent of the gamma of the holo$\left.\operatorname{gram}^{4}\right)^{5}$ ).

To fulfill the condition of eq. (8) in the strictest sense might require, according to eq. (4),

$$
\frac{1}{2}\left(E_{0}^{2}+E_{1}^{2}\right)>E_{0} E_{1}
$$

implying that the intensity in one of the beams must be somewhat different from the intensity of the other beam; this condition is readily achieved in practice (for instance, by different coatings of the mirrors in the two interferometer arms). We have shown, however, (See fig. 4-6), that spectra can be reconstructed with an interferometer in which both mirrors have the same reflectivity, because the effect of having the same intensity in the two beams, in holography, generally only results in the appearance of higher order "spectra", somewhat like the higher order spectra in quadratic detection in electrical engineering ${ }^{5}$ ), or indeed in optical gratings ${ }^{23}$ ). Further details of this analysis and detailed conditions are given in our ref. 22, together with additional experimental results, showing in particular highresolution spectra, which we have obtained by this method of holographic Fourier-transform spectroscopy.

Here, we may simply note ${ }^{4}$ ) that the relation between $H(x)$ and $I(x)$ may more generally be given in the form of a Taylor series expansion about the intensity $I_{0}$, in the form

$$
H(x)=H(I)=H\left(I_{0}\right)+H^{\prime}\left(I_{0}\right)\left(I-I_{0}\right)+\frac{H^{\prime \prime}\left(I_{0}\right)}{2 !}\left(I-I_{0}\right)^{2}+\ldots
$$

We may also wish to note here a dualism between the recording of spectral frequencies in holographic spectroscopy and the recording of spatial frequencies in (single-spectral frequency) holographic imaging, in particular in the case of spatially noncoherent sources ${ }^{9}$ ).

A discussion of the limitations, characteristic of holography, associated with the recordability of the interference-fringe components, with special application to spectroscopy, and a comparison of the "luminosity-resolution" parameters with other methods of spectroscopy, in view of singling out possible areas of application, will also be given in more detail in our ref. 22.

We may, however, give a very simple indication of the resolutions which are attainable by our method of holographic Fourier-transform spectroscopy. Let the hologram be recorded within an aperture $W$ along $x$ (see fig. 2). Let $a_{\lambda}$ be the period of the "interference grating" corresponding to the wavelength $\lambda$ in the hologram. According to the grating equation ${ }^{23}$ )

$$
\sin i+\sin i^{\prime}=m \frac{\lambda}{a}
$$

illumination of the hologram with a plane wave $\Sigma_{i}$ at the angle $i=0$, will 
produce a first order image at

$$
\left(\xi_{0}^{\prime}\right)_{1} \cong f \frac{\lambda_{L}}{a_{\lambda}}=f \lambda_{L} \nu_{\lambda}
$$

(see fig. 2), where $\lambda_{L}$ is the wavelength of the laser light, used in the reconstruction, and where we let $\nu_{\lambda}=1 / a_{\lambda}$ be the spatial frequency of the "interference grating" corresponding to the wavelength $\lambda$ in the hologram. By differentiation of eq. (13) it readily follows that

$$
\frac{\mathrm{d}\left(\xi_{0}^{\prime}\right)_{1}}{\mathrm{~d} \nu_{\lambda}}=f \lambda_{L}
$$

i.e.

$$
\Delta\left(\xi_{0}^{\prime}\right)_{1}=t \lambda_{L} \Delta \nu_{\lambda}
$$

We now further recall (see eq. 4) that the grating frequency $\boldsymbol{v}_{\lambda}$ is inversely proportional to $\lambda$. Let $C$ be the constant of proportionality. We have

$$
v_{\lambda}=\frac{C}{\lambda}
$$

and therefore, with eq. (15)

$$
\Delta\left(\xi_{0}^{\prime}\right)_{1}=-C / \lambda_{L} \frac{\Delta \lambda}{\lambda^{2}}
$$

According to the Rayleigh criterion, $\left.\left.\left.{ }^{4}\right)^{13}\right)^{14}\right)^{23}$ ) the spectral limit of resolution is obtained when (see fig. 2)

$$
\Delta\left(\xi_{0}^{\prime}\right)_{1}=f \frac{\lambda_{L}}{W}
$$

where, we recall, $W$ is the width of the hologram. In equating eq. (17) and eq. (18), and by recalling eq. (16), we finally have, for the resolving power of the holographic Fourier-transform spectroscopy method at the wavelength $\lambda$ (to which corresponds a hologram fringe frequency $v_{\lambda}$ ) the equation

$$
R . P . \equiv \frac{\lambda}{\Delta \lambda}=W \nu_{\lambda}=N
$$

where $N$ is the total number of fringes recorded on the hologram in the wavelength $\lambda$. The resolving power given by eq. (18) is recognized to be identical to that of a grating of width $\left.W^{23}\right)$. For ex., let $\nu_{\lambda}=2000$ fringes/ $\mathrm{mm}$ (readily recordable on KODAK 649F spectroscopic plates). The resolving power is then

$$
\text { R.P. }=2000 W(\text { per } \mathrm{mm} \text { of } W)
$$

A hologram as small as $W=20 \mathrm{~mm}$ (such as that of fig. 5) may therefore yield a resolving power of 40000 , with this fringe frequency. 
We may now note that the attainment of high resolutions may at first sight appear to present some problems, when an extended source aperture is used in the holographic interferometer. The use of extended source apertures (such as that which we used in our work shown in figures $4,5,6$ ) is of particular interest when very high luminosity is also of interest, even beyond that attainable in the basic form (small source aperture interferometric and Fourier-transform photoelectric spectroscopy) of which the high luminosity was first stressed by Jacquin ot $\left.\left.\left.{ }^{16}\right)^{17}\right)^{24}\right)^{25}$ ).

We have recently demonstrated ${ }^{10}$ ) ${ }^{11}$ ) an a posteriori compensation method, which permits to surmount such effects, which may result in a loss of resolution in holographic interferometers.

2. A posteriori, resolution-retrieving compensation of source effects $\left.{ }^{10}\right) \mathbf{1 1}$ ). When sources other than "point" sources are used in holography, either for the recording or for the reconstruction, the resolution of the image-forming process is reduced, because of a "spreading" or "smearing" of the image of a point-object. The resolution loss enters (as we have briefly shown in our ref. 10 and 11), in the form of a convolution between the object and the image of the source, so that we may say that the image of a point object is spread out to the width of the source image 4 ).

In the past, it had generally been assumed that the use of an "extended" rather than a "point" source, in the recording of a hologram, would result in an irretrievable loss of resolution.

The resolution "loss" which would result from an uncompensated "source effect" would be very considerable indeed, e.g. in $X$-ray holographic microscopy which we have been investigating for some time now, and where the smallest a vailable $X$-ray sources may not be less than some $100 \AA$ in diameter, for image formation at wavelengths where a $1 \AA$ resolution may be sought $\left.\left.{ }^{4-8}\right)^{10-14}\right)^{26}$ ). More generally, source effects enter in all holographic imaging.

A very important advance was made in holography, and indeed in image formation in coherent light, when Stroke e.a. showed ${ }^{10}$ ) ${ }^{11}$ ) that the "loss" of resolution, which would result from the recording of a hologram with an extended source, could, paradoxically, be retrieved, in the reconstruction, by illuminating the hologram also with an extended source provided that the correlation function of the two suitably structured sources had a narrow central peak, of a width comparable to the resolution limit sought in the two-step process. This is the case for structured sources having a broad spatial-frequency representation, for example, a Fresnel-zone plate, which can also be obtained by interference ${ }^{11}$ ).

A concise analysis of our holographic source compensation is given in our ref. 10 and 11, and a somewhat more detailed analysis in our ref. 4 and ref. 26. 
Here we merely wish to point to these references 10,11 , and 26 , because their results are indeed directly applicable to the method of holographic Fourier-transform spectroscopy which we described above.

The essence of the method can, however, be briefly stated as follows, because of the importance of the conclusion.

We may again consider as a model the one-dimensional Fourier-transform holography arrangements of figures 1 and 3 , which would normally use a point reference source $T_{s}(\xi)=\delta(0)$ at $R$. However, in place of the point reference, we now use a spatially coherent, extended source, of complex amplitude given by the equation

and an object

$$
T_{s}(\xi-a)
$$

$$
T_{0}(\xi)
$$

where $a$ is now taken as the usual "off-set" between the "centers of gravity" of $T_{s}$ and $T_{0}$. According to a procedure comparable to that used to obtain eq. (4) and eq. (6) we find $\left.{ }^{10}\right)^{11}$ ) that the hologram intensity is obtained under these conditions in the form

$$
I(x)=\left[t_{o} t_{o}^{*}+t_{s} t_{s}^{*}\right]+t_{o} t_{s}^{*} \exp (2 \pi i a x)+t_{o}^{*} t_{s} \exp (-2 \pi i a x)
$$

where $t_{o}$ and $t_{s}$ are the Fourier transforms of $T_{o}$ and $T_{s}$, respectively $\left.{ }^{4}\right)$.

Under the Fourier-transform reconstruction conditions of fig. 2 illumination of the hologram with a point source (to which corresponds the plane wave $\Sigma_{i}$ ) will produce an image, in which the two side-band images of interest are, respectively, the Fourier transforms of the complex amplitude transmittance corresponding to the second and third terms in eq. (22). By making use of the theory given in ref. 4, 13, 14, we obtain by Fourier transformation of amplitude transmittance in eq. (22), the equations

respectively

$$
\left[T_{o} * T_{s}^{*}\right]_{\xi-a}
$$

$$
\left[T_{o}^{*} * T_{s}\right]_{\xi+a}
$$

for the amplitudes in the upper-side-band, respectively lower-side-band images, where $*$ indicates a correlation, and where the image coordinate is taken as $\xi$, under unit magnification. The images are seen to be "spread" out, by correlation with the extended source $T_{s}$.

However, if we now illuminate the hologram, not with a point source, but with an "extended source" $T_{s^{\prime}}$ (which may or may not be equal to $T_{s}$ ), and if we let $t_{s^{\prime}}$ be the Fourier transform of $T_{8^{\prime}}$, then the complexamplitude transmission through the hologram, in the upper side band of eq. (22) becomes

$$
t_{s^{\prime}}\left[t_{o} t_{s}^{*} \exp (2 \pi i a x)\right]=\left[t_{o} \exp (2 \pi i a x)\right]\left[t_{s^{\prime}} t_{s}^{*}\right]
$$

The corresponding upper side-band image, obtained by Fourier transfor- 
mation of eq. (25) is equal to

$$
\left[T_{o} \otimes\left(T_{s^{\prime}} * T_{s}^{*}\right)\right]_{\xi-a}
$$

where $\otimes$ indicates a convolution. We note that, if

$$
\left[T_{s^{\prime}} * T_{s}^{*}\right]=\delta \text {, a delta function }
$$

then

$$
\left[T_{o} \otimes \delta\right]_{\xi-a}=\left[T_{o}\right]_{\xi-a}
$$

Eq. (27) states that the reconstructed upper side-band image will be identical to the object if the correlation of the reconstructing source with the complex conjugate of the recording source is equal to a delta function. Eq. (27) gives the condition under which the use of extended sources in holography will not result in any loss of resolution.

For instance, if $T_{s^{\prime}}$ is equal to $T_{s}$ then

$$
\left[T_{s} * T_{s}^{*}\right]
$$

is the auto-correlation function of the source. $\left[T_{s} * T_{s}^{*}\right]$ will be a function with a very narrow central peak, for example, if $T_{s}$ can be represented by a very broad spectrum of spatial frequencies. We may note, that such a

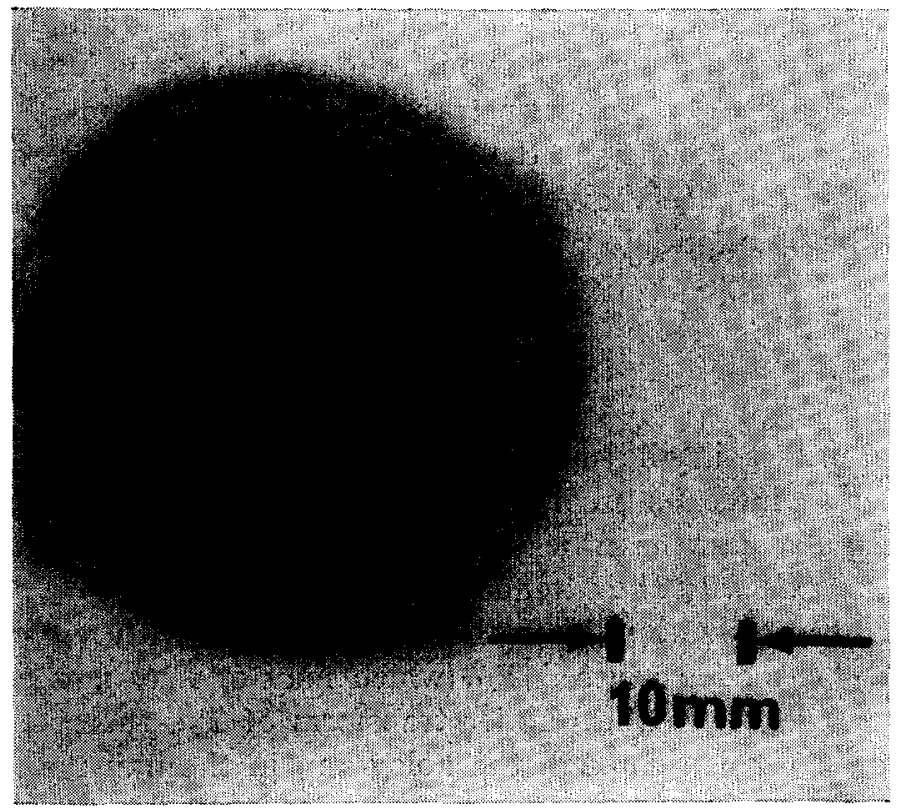

Fig. 7. "Coded" Fourier-transform hologram of word "Fourier" and of symbol " $\int \mathrm{d} \xi$ " (see fig. 8), recorded by using an extended, $10 \mathrm{~mm}$ diameter Fresnel-zone plate (maximum zone-frequency of 100 lines/mm, in the outermost zone) in place of the usual point source $R$ (see fig. 1), in the recording of the hologram $(f=600 \mathrm{~mm}$ ). 


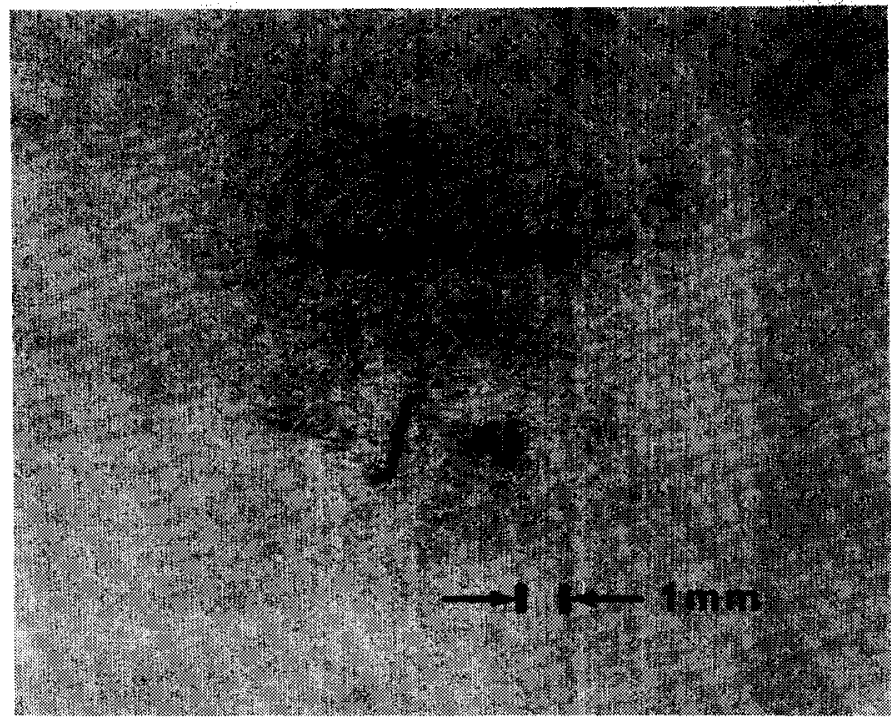

Fig. 8. Compensated image, reconstructed by Fourier transformation (see fig. 2) according to eq. (25) and eq. (26), by illuminating the "coded" hologram of fig. 7 by the same extended Fresnel-zone plate source, as that used in the recording (in place of the point source), in order to retrieve a posteriori ${ }^{10}$ ) ${ }^{11}$ ) the resolution, which would have been "lost" (because of a "spreading" of every image point into an extended area, that is into a $10 \mathrm{~mm}$ Fresnel-zone plate, in this case, according to eq. (23).

broad-spatial frequency spectrum representation would require an extended source field, so as to represent the various spatial frequencies with sufficient accuracy: such an extended source would therefore also result in a desirable great luminosity in the holographic system. We have already mentioned a Fresnel-zone plate as one example of such an "extended" structured source. ${ }^{10}{ }^{11}$ )

Examples of resolution retrieving compensation using these principles were first given by Stroke e.a. in our ref. 10 and 11 . However, an important previously unpublished example is shown in fig. 7 and fig. 8 . Figure 7 shows the hologram of the object, formed of the word "Fourier" and the symbol " $\int \mathrm{d} \xi$ " recorded with $T_{s}$ equal to a $10 \mathrm{~mm}$ diameter Fresnel-zone plate (itself previously recorded by interference) used in place of the usual point source in the recording. Fig. 8 shows the "compensated" image, reconstructed by using the same extended Fresnel-zone plate $T_{s^{\prime}}=T_{s}$, in place of the point source in the reconstruction. In comparing the $10 \mathrm{~mm}$ diameter of the Fresnel-zone plate used in the recording (and reconstruction) to the $1 \mathrm{~mm}$ scale of the image (fig. 8), it is apparent to what a remarkable degree "compensation" can be achieved. Compensated images of an even higher quality, obtained with a $19 \mathrm{~mm}$ "Fresnel half-zone plate" (the portion on one side of a diameter of the Fresnel-zone plate used here) are given in our ref. 11, where we have also noted analogies of our method of holographic a 
posteriori compensation, with the methods of a posteriori apodisation, described by Jacquinot and Roizen-Dossier ${ }^{27}$ ) in connection with quite different applications. We had already previously noted the possibilities of a posteriori compensation, using Fourier-transform computing methods, in connection with high-resolution spectroscopy using optical gratings ${ }^{28}$ ).

Conclusion. It should be clear that the methods of a posteriori "resolution-retrieving image compensation" and the holographic principles outlined above, and in the references 10,11 and 4, have applications not only in the special form of holographic Fourier-transform spectroscopy, which we describe. Improvements in performance of most all spectroscopic work should result from judicious application of holographic "compensation" methods, used a posteriori, or otherwise, especially when known instrumental limitations of resolution and luminosity must be surmounted, and may be surmounted, as it now appears possible.

\section{REFERENCES}

1) Gabor, D., Nature, 161 (1948) 777.

2) Gabor, D., Proc. Roy. Soc. (London) $\mathbf{1 9 7}$ (1949) 454.

3) Gabor, D., Proc. Roy. Soc. (London) B64 (1951) 449.

4) For a recent review of this field, see e.g. G. W. Stroke, "An Introduction to Coherent Optics and Holography", (Academic Press, New York and London, 1966).

5) Stroke, G. W. and Falconer, D. G., Phys. Letters, 13 (1964) 306.

6) Stroke, G. W. and Falconer, D. G., Phys. Letters, 15 (1965) 238.

7) Stroke, G. W., Appl. Phys. Letters 6 (1965) 201.

8) Stroke, G. W., Brumm, D. and Funkhouser, A., J. Opt. Soc. Amer. 55 (1965) 10.

9) Stroke, G. W. and Funkhouser, A., Phys. Letters, 16 (1965) 272.

10) Stroke, G. W., Restrick, R., Funkhouser, A. and Brumm, D., Phys. Letters 18 (1965) 274.

11) Stroke, G. W., Restrick, R., Funkhouser, A. and Brumm, D., Appl. Phys. Letters 7, (1965) 178 .

12) Gabor, D., Stroke, G. W., Restrick, R., Funkhouser, A, and Brumm, D., Phys. Letters 18 (1965) 116.

13) Stroke, G. W., "An Introduction to Optics of Coherent and Non-Coherent Electromagnetic Radiations", 1st Edition (The University of Michigan, May 1964).

14) Stroke, G. W., "An Introduction to Optics of Coherent and Non-Coherent Electromagnetic Radiations", 2nd Edition (The University of Michigan, March 1965).

15) Leith, E. N. and Upatnieks, J., J. Opt. Soc. Amer. 54 (1964) 1295.

16) e.g. Jacquinot, P., "New Developments in Interference Spectroscopy", in Reports on Progress in Physics, Vol. XXIII, (1960) p. 267-312.

17) Colloquium papers of "Les progrès récents en spectroscopie interférentielle", published in extenso in J. Physique, Radium 19 (1958) 185.

18) Connes, J., Rev. Optique 40 (1961) 45, 116, 171, 231.

19) J acquinot, P. and Dufour, Ch., J. Recherches C.N.R.S., labs. Bellevue (Paris) 6 (1948) 91.

20) Fellgett, P., Thesis, The University of Cambridge (1951).

21) Strong, J., J. Opt. Soc. Amer. 47 (1957) 354.

22) Stroke, G. W. and Funkhouser, A. (in preparation). 
23) Stroke, G. W., "Diffraction Gratings", in Handbuch der Physik, vol. 29, ed. S. Flügge (Springer Verlag, Berlin and Heidelberg, in print).

24) Jacquinot, P., in XVIIe Congrès du G.A.M.S., Paris (1954).

25) J a cquinot, P., J. Opt. Soc. Amer. (1958) 761.

26) Stroke, G. W., "Attainment of High Resolutions in Image-Forming X-ray Microscopy with 'Lensless' Fourier-Transform Holograms and Correlative Source Compensation", in Proceedings of Fourth International Congress of X-ray Optics (Held in Paris, 7-10 September 1965), (Hermann Editions, Paris, in print).

27) Jacquinot, P. and Roizen-Dossier, B., "Apodisation", in Progress in Optics, Vol. III ed. E. Wolf (North Holland Publishing Co. Amsterdam, 1964) pp. 31-188.

28) Stroke, G. W., "Ruling, Testing, and Use of Optical Gratings for High-Resolution Spectroscopy", in Progress in Optics, Vol II ed. E. Wolf (North-Holland Publishing Co., Amsterdam, 1963) pp. 1-72. 